\title{
Impact of antibiotics on the gut microbiota of critically ill patients
}

\section{Correspondence \\ Lorenzo Morelli \\ lorenzo.morelli@unicatt.it}

Received 9 May 2007

Accepted 7 April 2008

\author{
Gaetano lapichino, ${ }^{1}$ Maria Luisa Callegari, ${ }^{2}$ Silvia Marzorati, ${ }^{1}$ \\ Marco Cigada, ${ }^{1}$ Davide Corbella, ${ }^{1}$ Susanna Ferrari ${ }^{2}$ and Lorenzo Morelli ${ }^{3}$ \\ ${ }^{1}$ Istituto di Anestesiologia e Rianimazione, Università degli Studi, Polo San Paolo, via Di Rudinì 8,
I-20142 Milano, Italy \\ ${ }^{2}$ Centro Ricerche Biotecnologiche, Università Cattolica del Sacro Cuore, Via Milano 24, 26100 \\ Cremona, Italy \\ ${ }^{3}$ Istituto di Microbiologia, Università Cattolica del Sacro Cuore, Via E. Parmense 84, 29100 \\ Piacenza, Italy
}

\begin{abstract}
We evaluated the relationship between the intestinal microbiota composition and clinical outcome in a group of 15 high-risk patients admitted for acute infection and/or surgical/accidental trauma who were treated with systemic antibiotics according to standard intensive care unit (ICU) protocols. There was a high mortality rate amongst these patients, each of whom had a considerable organ failure score at admission, respiratory assistance during the most of their ICU stay and a long length of stay. All of these individuals received sedation and enteral nutrition, and the majority also received insulin, vasoactive drugs and some stress-ulcer prophylaxis agents.

The intestinal microbiota composition was assessed using denaturing gradient gel electrophoresis (DGGE), a molecular biology tool used to characterize bacterial ecosystems. As all of the patient subjects were in good health prior to their acute illness and admission to the ICU, the first faecal samples obtained from this group showed a DGGE banding pattern that was similar to that of healthy subjects. After 1 week of critical illness, coupled with intensive care treatment, including antibiotics, a very definite alteration in the overall microbiota composition was evident, as revealed by a reduction in the number of DGGE bands. Further pronounced changes to the DGGE banding profiles could be observed in patients remaining in the ICU for 2 weeks. Moreover, a dominant band, identified by sequencing as highly related to Enterococcus, was detected in the DGGE profile of some of our patient subjects. We also performed real-time PCR and obtained results that were in agreement with our qualitative evaluations using DGGE. The degree of organ failure and ICU mortality was significantly higher in patients for whom a high reduction in microbiota biodiversity was coupled with a massive presence of enterococci. $A$ statistically significant link between these two ecological traits and the use of clindamycin was also found.
\end{abstract}

\section{INTRODUCTION}

The human intestinal microbiota is a microbial community with a high biodiversity index that plays a key role in the maintenance of health (O'Hara \& Shanahan, 2006). The microbiota of a healthy subject prevents both virulence gene expression and exogenous bacterial colonization by competing with other micro-organisms for nutrients and binding sites on gut epithelial cells. Accordingly, the microbiota also prevents the onset of a cytokine-induced

Abbreviations: CLOS, critical length of stay; DGGE, denaturing gradient gel electrophoresis; ICU, intensive care unit; LOS, length of stay; SAPSII, simplified acute physiology score II; SOFA, sequential organ failure analysis; TGGE, temperature gradient gel electrophoresis. intestinal barrier permeability in response to adhering pathogenic bacteria (Guarner \& Malagelada, 2003; Marshall, 1999; Alverdy et al., 2003). Under normal conditions, the composition of this microbial ecosystem is quite stable. However, human microbiota can be negatively affected by external factors, and these include antibiotic treatments (Sullivan et al., 2001; Bartosch et al., 2004).

A number of factors that predispose the gut ecology to alterations can occur during the care of critically ill patients. Those that have been described include: luminal hypoxia and hypercarbia; the use of agents to neutralize gastric secretions; vasoactive agents; sedation and analgesia that may impair intestinal motility; nutrient scarcity within 
the colon due to parenteral or enteral diets poor in nondigestible carbohydrates (Schneider et al., 2000); and severe sepsis/injury or digestive surgical stress (Marshall, 1999; Alverdy et al., 2003). It is thus highly possible that disruptions to the ecological balance of the intestinal microbial community could occur in critically ill patients undergoing antibiotic treatments.

It is difficult to draw a full and accurate picture of the microbiota in the intestinal tract, as the gut contains more than 600 different bacterial species, most of which cannot be cultured under laboratory conditions (Guarner, 2006). However, culture-independent techniques to investigate microbial gut ecology are now available (Tannock, 2002) and allow better monitoring of these intestinal communities and also of population shifts. Despite the potential relevance of the disruption of the gut microbial ecology to the final clinical outcomes in critically ill patients, there are few studies on this topic (Alverdy et al., 2003; Shimizu et al., 2006). The aim of our current observational study was to assess, by means of molecular techniques such as denaturing gradient gel electrophoresis (DGGE), faecal microbiota changes in high-risk, critically ill patients during their intensive care unit (ICU) stay and treatment with antibiotics, according to the standard ICU protocols.

\section{METHODS}

Study group. Between February and June of 2006, a prospective, observational cohort study was designed to study faecal microbiota variations in consecutive cases of critical illness in the adult general ICU of a university hospital. Patients with acute organ failure and who were expected to need mechanical ventilation for at least 4 days (critical length of stay: cLOS) were enrolled. Exclusion criteria included a hospital stay and/or antibiotic treatment prior to ICU admission, opportunistic/autoimmune diseases, and cancer or steroid therapies. The patient characteristics, diagnosis, simplified acute physiology score II (SAPS II) (Le Gall et al., 1993) and sequential organ failure assessment (SOFA) score (Vincent et al., 1996) were collected for each individual at ICU admission. Treatments, diarrhoea, SOFA, length of ICU stay (LOS), cLOS and vital status at ICU/hospital discharge were also recorded. Treatments recorded included: ventilation, the type and amount of nutrition, insulin, systemic/selective-digestive antibiotics (tobramycin, colistin and amphotericin B), vasoactive drugs, agents neutralizing gastric secretions and lactulose. Diarrhoea was defined as at least three discharges exceeding, in total, $400 \mathrm{ml}$ per day (Bliss et al., 1992). Due to the observational structure of this study, no control groups were included.

Microbiota analysis. For each patient, faeces were collected at ICU admission (T0) and then weekly (T1, T2, etc.) from a rectal sinus, thus avoiding collection by enema, and were stored at $-20{ }^{\circ} \mathrm{C}$ until DNA analysis. Patients were excluded if the T0 or T1 samples could not be harvested. The presence of Clostridium difficile toxin in the diarrhoeal stools was also assayed using ImmunoCard Toxins A\&B (Meridian Bioscience). Total bacterial DNA extractions were performed with $200 \mathrm{mg}$ faecal material using the PSP Spin Stool DNA kit (Invitek) according to the manufacturer's instructions. Target DNA was amplified using commercially available Megamix (Microzone) and a Perkin Elmer thermal cycler 9700. PCR amplifications of total bacterial DNA were obtained using the primer pair HDA1-GC and HDA2, and the thermocycling program as described previously (Walter et al., 2000).

DGGE analysis was performed with a DGene System (Bio-Rad Hercules) using a $0.8 \mathrm{~mm} 6 \%$ polyacrylamide gel with a ratio of acrylamide to bisacrylamide of $37.5: 1$ and containing a $35-55 \%$ gradient of urea and formamide, as reported previously (Walter et al., 2000). The electrophoretic run was performed in 1× Tris/acetate/ EDTA buffer (TAE) at $120 \mathrm{~V}$ for $5 \mathrm{~h}$. DGGE gels were stained for 30 min with SYBR Green I (Roche Applied Science) in 1× TAE buffer. The DGGE profiles obtained for each sampling were then compared within each gel using Fingerprint II software (Bio-Rad Hercules). These analyses included the calculation of the number of bands for each pattern and comparisons between patterns using the Pearson coefficient as a measure of the degree of similarity (Seksik et al., 2003). Two identical profiles create a similarity value of $100 \%$ whereas completely different profiles result in a similarity value of $0 \%$.

To identify the bacteria producing the DNA banding pattern by DGGE at the species level, bands were excised from the stained gels with a sterile scalpel and eluted overnight in $20 \mu \mathrm{l}$ sterile water at $4{ }^{\circ} \mathrm{C}$, as described by Ben Omar \& Ampe (2000). One microlitre of the eluted DNA from each band was then used as the template for a PCR reaction under the conditions described above. The resulting PCR products were purified using a Wizard SV Gel and PCR clean-up system (Promega). The final eluents, containing the purified PCR fragments, were sequenced by BMR Bio Molecular Research (CRIBI, Padova, Italy). The closest known relatives to the partial 16S rRNA gene sequences obtained were identified using the BLAST and RDP programs.

The percentage similarities between the DGGE profiles at T0 and at the subsequent sampling times for each patient were used as an index of microbiota modifications. A value equal or higher than the median value of similarity for all of the T1 profiles, compared with the baseline, was defined as the index of microbiota biodiversity preservation. This index value, coupled with the appearance in some samples of a new dominant DNA band (which was identified as Enterococcus spp.), was then evaluated against clinical variables.

Quantification of Enterococcus by real-time PCR. We quantified the presence of Enterococcus genomic DNA in the patient faecal samples by real-time PCR. The reactions were performed using the LightCycler System and LightCycler FastStart DNA Master ${ }^{\text {PLUS }}$ SYBR Green I (Roche Applied Science). The fragment of the 16S rRNA gene specific for the Enterococcus genus was amplified using the primers ECF (AGAAATTCCAAACGAACTTG) and ECR (CAGTGCTCTACCTTCCATCATT) (He \& Jiang, 2005). The $20 \mu$ total reaction volumes contained $4 \mu$ l LightCycler FastStart DNA Master ${ }^{\text {PLUS }}$ SYBR Green I (Roche Diagnostics), $0.5 \mu \mathrm{M}$ forward and reverse primers and $5 \mu \mathrm{l}$ extracted DNA. The amplification program included an initial denaturation step at $95{ }^{\circ} \mathrm{C}$ for $10 \mathrm{~min}$, followed by 40 cycles of denaturation for $15 \mathrm{~s}$ at $95{ }^{\circ} \mathrm{C}$, annealing for $20 \mathrm{~s}$ at $63{ }^{\circ} \mathrm{C}$, and extension for $30 \mathrm{~s}$ at $72{ }^{\circ} \mathrm{C}$. The temperature transition rate was $20{ }^{\circ} \mathrm{C} \mathrm{s}^{-1}$.

For melting curve analysis, the samples were denatured at $65{ }^{\circ} \mathrm{C}$ for $15 \mathrm{~s}$, and then slowly heated to $95{ }^{\circ} \mathrm{C}$ with a temperature transition rate of $0.1{ }^{\circ} \mathrm{C} \mathrm{s}^{-1}$ and a continuous monitoring of fluorescence. Enterococcus faecium DNA (BAA-472D-5) was purchased from the American Type Culture Collection and used to optimize the real-time reactions and generate quantification standards. For the construction of standard curves, a 10 -fold dilution series ranging from $0.01 \mathrm{pg}$ to $10 \mathrm{ng}$ of the target species genomic DNA was used. The standard curves had correlation coefficient values of between 0.99 and 1.0, whereas the amplification slope was between -3.35 and -3.38 . The standard curves for the real-time PCR assays were used to quantify the target bacterial DNA from faecal DNA preparations and PCR 
results were converted to a mean estimate of the target bacterial genomes present in $1 \mathrm{~g}$ faeces (wet wt). The estimated mean genome size for Enterococcus that was used for converting the results obtained by real-time PCR was $3 \mathrm{Mb}$. Three parallel PCR reactions for each sample were analysed. The mean amounts and standard deviations of the target genomes in $1 \mathrm{~g}$ faeces, calculated by PCR, are shown in Table 3 .

Statistics. Categorical variables were compared using the Fisher exact test. Continuous variables were compared with the Student's $t$-test for normally distributed variables, the Mann-Whitney test for those that were abnormally distributed, or with simple-multiple linear regressions. The results are expressed as the mean $\pm \mathrm{SD}$ or median (25th-75th percentile) when appropriate. The level of significance was set at $P<0.05$. Statistical analyses were performed using the Intercooled Stata 8.0 statistical package.

\section{RESULTS AND DISCUSSION}

\section{Clinical course}

In our current study, we evaluated the relationship between the intestinal microbiota composition and clinical outcomes in a select group of high-risk patients who presented with acute infection ( $40 \%$ had full-blown septic shock) or surgical/accidental trauma, resulting in high severity of illness/organ failure score at admission (Table 1). Thirteen males (37-79 years) and two females (59-66 years) out of the 30 eligible patients included in our study cohort completed the faecal sample collection protocol until T1. During this period, each patient required respiratory assistance and received systemic/local antibiotics, sedation and, by the second day, enteral nutrition without fibre enrichment (mean $985 \pm 330 \mathrm{kcal}$ per day) (Table 2). Thirteen patients received insulin, six were given vasoactives, four received agents to neutralize gastric secretions and two were administered lactulose. Nine patients in this

Table 1. Characteristics of the patients in the current study cohort at the time of ICU admission

\begin{tabular}{|lcclcc|}
\hline No. & Sex & Years & Diagnosis & SAPS II & SOFA \\
\hline 1 & M & 64 & Peritonitis-shock & 43 & 7 \\
2 & M & 74 & Pneumonia & 42 & 5 \\
3 & M & 76 & Pneumonia & 39 & 6 \\
4 & M & 73 & Severe bronchitis & 53 & 3 \\
5 & M & 60 & Pneumonia & 45 & 5 \\
6 & M & 79 & Pneumonia & 35 & 7 \\
7 & M & 78 & Pneumonia-shock & 59 & 8 \\
8 & M & 64 & Acute on COPD & 31 & 3 \\
9 & F & 59 & Pneumonia & 57 & 7 \\
10 & M & 75 & Severe bronchitis & 68 & 5 \\
11 & M & 39 & Cellulitis-shock & 47 & 14 \\
12 & M & 69 & Pneumococcus & 37 & 8 \\
& & & meningitis & & \\
13 & M & 37 & Politrauma & 23 & 3 \\
14 & F & 66 & Pneumonia-shock & 38 & 7 \\
15 & M & 79 & Peritonitis & 50 & 6 \\
\end{tabular}

group $(1,2,4,5,6,8,11,12$ and 14) had at least one episode of diarrhoea (Table 2). The seven patients who remained in the ICU after T1 had their antibiotic treatments modified according to their clinical situation, and the enteral nutrition support was set to full strength. In addition, three new patients $(7,13$ and 15$)$ showed an episode of diarrhoea. All 12 patients with diarrhoea were found to be negative for $C$. difficile toxin. Further faecal samples were also taken from one patient (14) after T2 (see case report).

\section{Microbiota analysis and clinical impact}

Few reports currently exist on the alterations to the gut microbiota that occur during a severe stress reaction (Shimizu et al., 2006), although more data are available on the effects of artificial nutrition (Schneider et al., 2000; Hebuterne \& Schneider, 2001) and antibiotic treatment (Sullivan et al., 2001; Bartosch et al., 2004). Hence there were many possible factors that predisposed our current patient cohort to an alteration of their gut ecology. It is also essential to point out that these patients were, from an intestinal microbiota perspective, in good health prior to the onset of their acute illness and subsequent ICU admission. The faecal microbiota of healthy subjects, when analysed by DGGE or temperature gradient gel electrophoresis (TGGE), shows complex, unique and individual profiles (Zoetendal et al., 2001; Tannock, 2002; Donskey et al., 2003; Mai \& Morris, 2004). Moreover, a report by Seksik et al. (2003) has shown that such molecular profiles of faecal microbiota are very stable over time (up to 1 year) under healthy conditions.

In our current study, we performed DGGE analysis of faecal samples in all of our patients at ICU admission and found, except for patient 14, a complex banding pattern (Fig. 1a, b) which included a number of bands previously described in healthy adults using the same technique (Seksik et al., 2003). The similarity between the DGGE profiles in each patient when comparing the $\mathrm{T} 1$ versus $\mathrm{T} 0$ samples was $62.06 \pm 27.11 \%$, median $68 \%$ (Table 2). By univariate analysis, the variation in the faecal microbiota at $\mathrm{T} 1$ was related to the degree of organ failure (SOFA: $R^{2}=0.43$, $P=0.011$ ) and complexity of ICU treatment (cLOS: $R^{2}=0.38, P=0.015$ ). The multivariate equation that we used to describe these relationships was: percentage similarity of DGGE profiles $=139.9-3.8$ SOFA (T1) -8.8 cLOS (T1) $\left(R^{2}=0.60, P=0.007\right)$. The median of the similarity of the DGGE profiles after one week was used to divide the cases into two groups with remarkably different clinical outcomes; the seven patients showing major modifications of their DGGE profiles had a higher SOFA score at T1 $(5.4 \pm 2.6$ vs $2.5 \pm 2.2 ; P=0.029)$, longer period of invasive ICU support (cLOS: $24.6 \pm 25.2,16,10-28$ vs $7.9 \pm 4.1,8,4.5-11$; $P=0.0174)$ and ICU LOS $(25.1 \pm 25.9,16,11-28$ vs $10.3 \pm 3.3,10.5,7.5-12.0 ; P=0.0315)$, coupled with a higher mortality ( 4 out of 7 vs 0 out of $8 ; P=0.026$ ). Moreover, although not statistically significant, the patients with 
Table 2. Antibiotic therapies, microbiota variations, SOFA, time period of diarrhoea onset, critical and whole ICU stay, hospital (HOS) stay, and outcomes for the indicated patients

\begin{tabular}{|c|c|c|c|c|c|c|c|c|}
\hline Patient & Antibiotics* & $\begin{array}{l}\text { Similarity of } \\
\text { DGGE profiles } \\
\text { T1 vs T0 }(\%)\end{array}$ & SOFA T1 & $\begin{array}{c}\text { Similarity of } \\
\text { DGGE profiles } \\
\text { T2 vs T0 (\%) }\end{array}$ & SOFA T2 & $\begin{array}{l}\text { Time period of } \\
\text { diarrhoea onset }\end{array}$ & cLOS & ICU/HOS stay \\
\hline 1 & CTX, CLI, SDD & 88.0 & 7 & - & & $\mathrm{T} 0-\mathrm{T} 1$ & 9 & $12 / 25$ \\
\hline $2 \dagger$ & AMP, CTX, CLI, SDD & 25.0 & 7 & - & & T0-T1 & 10 & $10 / 10$ \\
\hline 3 & CRO, CLR & 75.0 & 2 & - & & & 2 & $6 / 38$ \\
\hline 4 & CTX, CLR, SDD & 93.1 & 1 & - & & T0-T1 & 4 & $7 / 13$ \\
\hline $5 \dagger$ & CTX, CLR, SDD & 63.0 & 5 & - & & T0-T1 & 13 & $13 / 13$ \\
\hline $6 \dagger$ & CFZ, CLI & 62.9 & 2 & 57.4 & 11 & T0-T1 & 16 & $16 / 16$ \\
\hline $7 \dagger$ & CRO, CLR & 34.4 & 7 & 28.0 & 5 & $\mathrm{~T} 1-\mathrm{T} 2$ & 28 & $28 / 28$ \\
\hline 8 & CRO, CLR & 90.0 & 2 & - & & T0-T1 & 7 & $9 / 12$ \\
\hline 9 & CRO, CLR & 68.0 & 3 & - & & & 5 & $8 / 9$ \\
\hline 10 & CRO, CLI & 49.9 & 2 & - & & & 9 & $11 / 22$ \\
\hline 11 & $\begin{array}{l}\text { CRO, CLR, CLI, OXA, } \\
\text { SDD }\end{array}$ & 33.3 & 9 & 52.2 & 6 & T0-T1 & 16 & $18 / 33$ \\
\hline 12 & AMP, CRO & 84.7 & 1 & 39.4 & 0 & T0-T1 & 11 & $12 / 18$ \\
\hline 13 & CFZ & 82.2 & 1 & 51.2 & 1 & $\mathrm{~T} 1-\mathrm{T} 2$ & 11 & $12 / 22$ \\
\hline 14 & CRO, CLR, CLI & 3.8 & 6 & 10.5 & 7 & T0-T1 & 80 & $80 / 156$ \\
\hline 15 & CTX, CLI, SDD & 77.6 & 3 & 48.7 & 3 & T1-T2 & 14 & $16 / 26$ \\
\hline
\end{tabular}

*AMP, ampicillin; CFZ, cefazolin; CLI, clindamycin; CLR, clarithromycin; CRO, ceftriaxone; CTX, cefotaxime; OXA, oxacillin; SDD, selective digestive decontamination.

$\dagger$ Died. All deaths were in the ICU.

Table 3. Antibiotic therapies and microbiota variations in the patient cohort in this study

Results of RT-PCR are expressed as the amounts of bacterial target genomes of Enterococcus ( $\mathrm{g}$ faeces) ${ }^{-1}$.

\begin{tabular}{|c|c|c|c|c|c|c|}
\hline Patient & Antibiotics $^{*}$ & Enterococci in T0 & $\begin{array}{c}\text { Similarity of } \\
\text { DGGE profiles } \\
\text { T1 vs T0 (\%) }\end{array}$ & Enterococci in T1 & $\begin{array}{c}\text { Similarity of } \\
\text { DGGE profiles } \\
\text { T2 vs T0 (\%) }\end{array}$ & Enterococci in $\mathrm{T} 2$ \\
\hline 1 & CTX, CLI, SDD & $3.0 \mathrm{E}+6$ & 88.0 & $4.0 \mathrm{E}+9$ & - & \\
\hline $2 \dagger$ & AMP, CTX, CLI, SDD & $1.5 \mathrm{E}+5$ & 25.0 & $6.0 \mathrm{E}+10$ & - & \\
\hline 3 & CRO, CLR & $9.0 \mathrm{E}+7$ & 75.0 & $4.45 \mathrm{E}+7$ & - & \\
\hline 4 & CTX, CLR, SDD & $6.7 \mathrm{E}+6$ & 93.1 & $7.6 \mathrm{E}+6$ & - & \\
\hline $5 \dagger$ & CTX, CLR, SDD & $2.0 \mathrm{E}+6$ & 63.0 & $9.0 \mathrm{E}+10$ & - & \\
\hline $6 \dagger$ & CFZ, CLI & $6.7 \mathrm{E}+6$ & 62.9 & $1.3 \mathrm{E}+8$ & 57.4 & $5.6 \mathrm{E}+8$ \\
\hline $7 \dagger$ & CRO, CLR & $1.2 \mathrm{E}+7$ & 34.4 & $3.4 \mathrm{E}+8$ & 28.0 & $5.1 \mathrm{E}+9$ \\
\hline 8 & CRO, CLR & $4.4 \mathrm{E}+6$ & 90.0 & $3.0 \mathrm{E}+6$ & - & \\
\hline 9 & CRO, CLR & $7.8 \mathrm{E}+6$ & 68.0 & $2.6 \mathrm{E}+6$ & - & \\
\hline 10 & CRO, CLI & $5.0 \mathrm{E}+6$ & 49.9 & $2.3 \mathrm{E}+8$ & - & \\
\hline 11 & $\begin{array}{l}\text { CRO, CLR, CLI, OXA, } \\
\text { SDD }\end{array}$ & $7.5 \mathrm{E}+6$ & 33.3 & $3.0 \mathrm{E}+7$ & 52.2 & $1.2 \mathrm{E}+9$ \\
\hline 12 & AMP, CRO & $1.2 \mathrm{E}+6$ & 84.7 & $1.5 \mathrm{E}+6$ & 39.4 & $3.0 \mathrm{E}+7$ \\
\hline 13 & $\mathrm{CFZ}$ & $1.2 \mathrm{E}+7$ & 82.2 & $3.0 \mathrm{E}+7$ & 51.2 & $2.0 \mathrm{E}+7$ \\
\hline 14 & CRO, CLR, CLI & $8.5 \mathrm{E}+7$ & 3.8 & $4.5 \mathrm{E}+9$ & 10.5 & $7.9 \mathrm{E}+10$ \\
\hline 15 & CTX, CLI, SDD & $1.2 \mathrm{E}+6$ & 77.6 & $3.0 \mathrm{E}+5$ & 48.7 & $6.0 \mathrm{E}+8$ \\
\hline
\end{tabular}

*AMP, ampicillin; CFZ, cefazolin; CLI, clindamycin; CLR, clarithromycin; CRO, ceftriaxone; CTX, cefotaxime; OXA, oxacillin; SDD, selective digestive decontamination.

$\dagger$ Died. All deaths were in the ICU. 
(a)

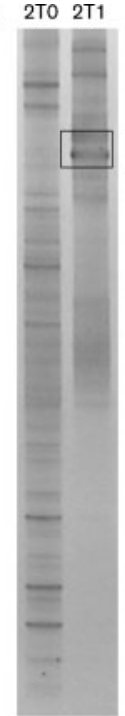

(b)
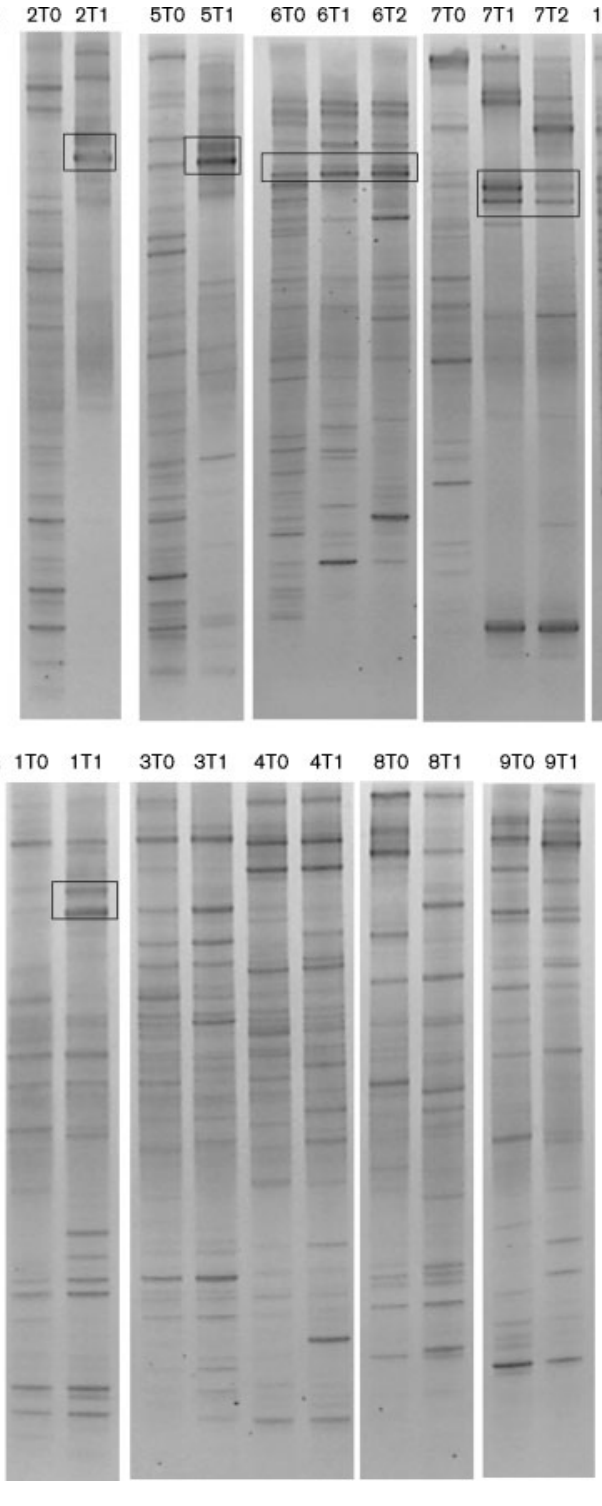
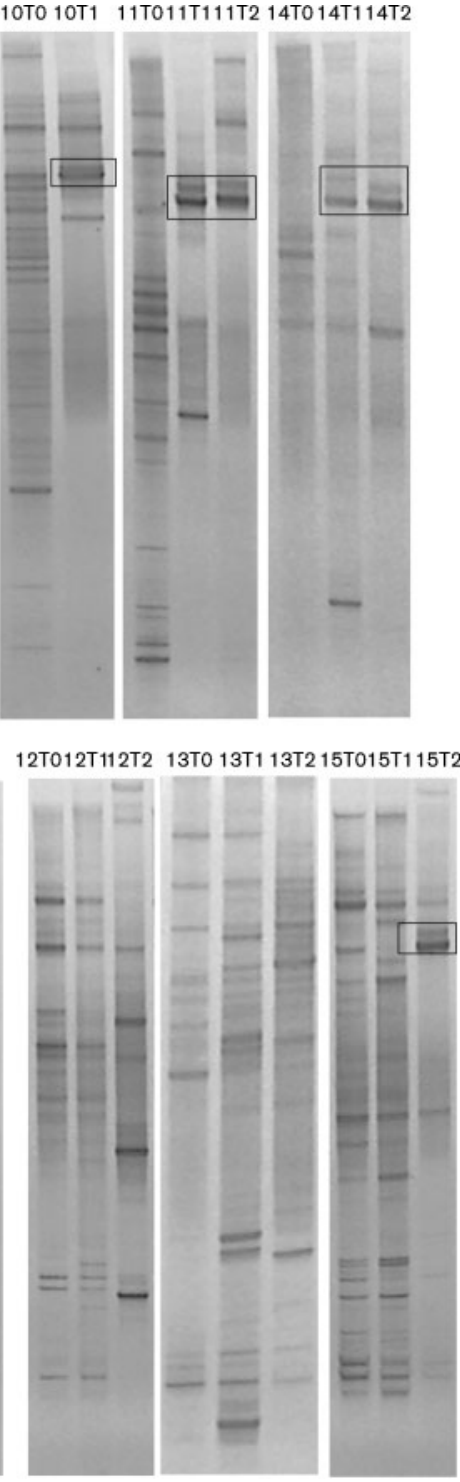

Fig. 1. DGGE analysis of antibiotic-dependent alterations of faecal microbial profiles. Bacterial community profiles were generated from faecal DNA using the universal PCR primers HDA1-GC/HDA2 and analysed in a $35-55 \%$ gradient gel. (a) DGGE profiles of the V2-V3 regions of the 16S rRNA genes in the faecal microbiota of patients showing a similarity value of $\mathrm{T} 1$ versus $\mathrm{T} 0$ that was lower than the median value. (b) DGGE profiles obtained from faecal samples of patients showing a similarity value of $\mathrm{T} 1$ versus $\mathrm{TO}$ that was equal or higher than the median value. Bands denoted by the black rectangles were excised and identified by sequencing as being strongly related to Enterococcus spp. greater DGGE profile changes received more anti-anaerobe drugs $(71.4 \%$ vs $37.5 \%)$.

Further alterations in DGGE profile were noted between T1 and $\mathrm{T} 2$, as demonstrated by the lower percentage similarity of the profiles observed between $\mathrm{T} 0$ and $\mathrm{T} 2$ for each patient compared with that seen between T0 and T1 (mean $41.1 \pm 16.6 \%$, $n=$ seven patients). As a result, over the entire ICU stay, only five patients $(1,3,4,8$ and 9) did not experience a reduction in the number of DNA bands in their faecal samples. These results suggest that a single week of critical illness, coupled with intensive ICU treatment, resulted in a definite alteration of the overall microbiota composition, which was evidenced by a reduction in the number of DGGE bands in the faecal samples from most of the patients. It is noteworthy in this regard that TGGE banding profiles of healthy subjects have been shown to have a $93 \%$ similarity index after 2 years (Seksik et al., 2003). Moreover, greater alterations in the DGGE banding profiles could be detected in patients who remained in the ICU for 2 weeks. None of the available variables was found to be capable of predicting the onset of diarrhoea (data not shown).

The second interesting finding from these analyses was that only 1 week of ICU treatment could cause the appearance of a very intense DGGE band which was faint or absent in the T0 profiles of the same subjects (Fig. 1). Sequence analysis of these bands, excised from the gels for all patients showing this variation, subsequently revealed a significant (more than 95\%) similarity with the sequences of the 16S rRNA gene of the Enterococcus genus. At T2, this Enterococcus band was still present in five patients out of the seven sampled.

As DGGE can only provide a qualitative estimate of the levels of a specific bacterial group, we used RT-PCR to perform more quantitative analysis of bacteria of interest 
(based on the DNA bands that were detected). The same approach was used previously by Rinttila et al. (2004), who showed that RT-PCR could result in an overestimation when compared with plate counting. The results that we present herein probably suffer from the same overestimation rate, but the relevant outcomes are the comparisons between the sampling points. Rinttila et al. (2004) employed RT-PCR for the quantification of Enterococcus from faecal samples of healthy volunteers and found that the number of bacterial genomes in $1 \mathrm{~g}$ faeces ranged from $10^{6}$ to $10^{7}$. The levels of Enterococcus target genomes (g faeces) $)^{-1}$ calculated in our present study are presented in Table 3. Nine of the patients considered in this study exceeded the upper limit.

The increase in the presence of Enterococcus bacteria among the gut faecal microbiota of our patients during their whole ICU stay could only be associated with the overall disruption to the microbiota by univariate analysis ( $\%$ of similarity of DGGE profiles vs T0 $<68 \%, P=0.006$ ) and with the clindamycin treatments $(P=0.006)$ that were administered in cases of ab ingestis pneumonia prophylaxis, peritonitis and severe cellulitis. The role of clindamycin in promoting overgrowth of enterococci has been previously shown by Jernberg et al. (2005). It should be noted also that Enterococcus is an emerging genus in ICU epidemiology (Weber \& Gold, 2003).

The loss of intestinal bacteria, particularly anaerobes, can induce an overgrowth of Enterococcus, a normal component of the human microbiota (Stiefel et al., 2004; Donskey, 2004). Moreover, a decrease in the number of gut anaerobes impairs the colonic degradation of undigested carbohydrates, provided via enteral nutrition, to absorbable lactic acid and short-chain fatty acids. This leads to an increased osmotic load. Reduced availability of butyrate, an important source of energy for the colonic mucosa (Sheppach et al., 1997), causes functional disorders (Topping \& Clifton, 2001) resulting in diarrhoea (Young \& Schmidt, 2004). Interestingly, as an indirect sign of severe alteration of bacterial gut ecology, $80 \%$ of the patients in our current cohort experienced at least one diarrhoea episode during enteral nutrition and antibiotic treatment.

Since an evaluation of this group of patients as a whole would make it difficult to draw any conclusions, we adopted a 'case by case' approach and focused our attention on individual patients whose index of microbiota biodiversity preservation was under the cut-off (Tables 1, 2 and 3; Fig. 1). We considered patients 5, 6 and 10 to be characterized by a minor ecological alteration (50-63\% similarity, DGGE profile vs basal profile) after a 1 week ICU stay. Bands later identified as Enterococcus were found to be present in each of these cases at a later stage in the ICU. Patients 5 and 6 had been admitted for severe respiratory failure due to pneumonia and later died. Both were responsive to intensive treatment as shown by their clinical condition coupled with a stable or improved SOFA score at T1. After T1, patient 5 worsened (SOFA score increased to 7) and died of septic shock before T2. Of note in this regard, enterococci were shown to have massively accumulated from T0 to T1 in this patient. Patient 6 remained clinically and ecologically stable until $\mathrm{T} 2$, and then had a brisk septic episode (positive blood culture for Staphylococcus aureus) with huge irreversible impairment of the lung and the development of cardiovascular conditions (SOFA 11). The elevated levels of Enterococcus, as detected by RT-PCR, occurred from T0 to $\mathrm{T} 1$ and approached a $2-\log$ increase.

In contrast, patient 10 was admitted for a severe diffuse bronchitis and an initial cardiac failure but survived. This individual showed a discrepancy between a very high severity and low organ failure score. This is characteristic of the bias that can arise with severity scores that give excess weight to old age and comorbidities. This male patient showed greater ecological alterations than the two deceased patients (a $50 \%$ similarity and just over the upper 'normal' limit for Enterococcus content), but also showed consistent improvements in his clinical condition (SOFA $\mathrm{T} 1=2$ ); he was transferred to the main wards of the hospital within 2 weeks of admittance to the ICU and before the collection of the T2 sample.

A further scenario that we investigated involved patients 2, 7, 11 and 14, who showed huge biota alterations with the percentage similarity of profiles observed well below the median value, and a massive Enterococcus content after 1 week in the ICU. Patients 2 and 7, who were admitted for pneumonia with severe respiratory failure, had high severity and organ failure scores and subsequently died. The clinical course of the illness in both cases was a slow progressive worsening, as shown by their high SOFA scores at the sampling times and just before death ( 7 for both). Accordingly, the alterations in the DGGE profiles and enterococci appearance remained very large, even during the second week for patient 7 , who died after T2.

Patients 11 and 14 showed the same reduced biota profile at T1 as patients 2 and 7, but survived. Patient 11 was a young man who developed septic shock as a result of extensive cellulitis from the right knee to the axilla. At admission, he showed a high severity (young, no comorbidities) and a huge organ failure score (14, about a $60 \%$ predicted probability of ICU death). The clinical condition of this patient progressively improved, however, and the SOFA decreased. In addition, the number of detectable bands on the DGGE gel increased from T1 to T2, and the enterococci levels showed an increase from the basal values. This patient was transferred to the main wards after 18 days in the ICU. Patient 14 was a 66 -year-old woman admitted to our ICU for septic shock due to bilateral Pneumococcus pneumonia after 7 days of neglected illness at home. This was associated with a reduction in the number of DGGE bands at ICU admission, suggesting that her gut microbiota was already altered, and the enterococci levels were calculated to be $8 \times 10^{7}$ genomes (g faeces) ${ }^{-1}$. The severe respiratory insufficiency of this patient was 
further complicated by mediastinitis, which resulted from a tracheal lesion during a difficult rescue intubation. The extensive lung consolidation and a long period of pulmonary septic capillary leak resulted in a refractory pulmonary hypertension that required 156 days of intensive/subintensive stay before a referral to another hospital for rehabilitation (Giacomini et al., 2007). The combination of severe lung infection, mediastinitis with systemic sepsis and full antibiotic treatment over 1 week was associated with an almost total disappearance of gut microbiota, which to a large extent was due to the outgrowth of enterococci. This critical, septic phase lasted for many of the ICU days for this patient and we collected faecal samples for 5 weeks and then on day 58 , when the clinical conditions seemed to advise a positive outcome. However, the DGGE profile of the gut microbiota of patient 14 showed a slow progressive increase in the number of bands from a T1 value of similarity of $3.8 \%$ to T3 (17.9\%: SOFA 7), T4 (18.5\%: SOFA 7), day 39 (16.8\%: SOFA 5) and day 58 (43.0\%: SOFA 7). The enterococci counts remained in the high range at $\mathrm{T} 1$ and $\mathrm{T} 2(6.6 \mathrm{E}+9$, $1.7 \mathrm{E}+10,1.1 \mathrm{E}+10,2.0 \mathrm{E}+10)$.

We conclude from our analyses that clinical improvements correspond to a progressive, if slow, enrichment of the faecal microbiota, even if the timing of the sampling may have obscured the evidence of this phenomenon. The corollary of this is that the microbiota remains reduced in number if clinical conditions do not improve for the patient.

The small sample size is a clear limitation of this study. However, for the first time, to our knowledge, we have shown that ecological alterations in gut microbiota in critically ill patients can be detected using molecular tools and that these data have clinical significance. Our findings underline: (a) the impact of even a single week of critical illness on the maintenance of the gut ecosystem and its negative influence on clinical outcome, and (b) the specific risk of Enterococcus outgrowth following both gut microbiota changes and clindamycin treatments. Moreover, these alterations, coupled with the effects of increased enterococci levels, seem to be associated with organ failure, the duration of intensive care, as well as the negative outcomes for the four patients in our current study cohort who died in the ICU.

\section{REFERENCES}

Alverdy, J. C., Laughlin, R. S. \& Wu, L. (2003). Influence of the critically ill state on host-pathogen interactions within the intestine: gut-derived sepsis redefined. Crit Care Med 31, 598-607.

Bartosch, S., Fite, A., Macfarlane, G. T. \& McMurdo, E. T. (2004), Characterization of bacterial communities in feces from healthy elderly volunteers and hospitalized elderly patients by using real-Time PCR and effects of antibiotic treatment on the fecal microbiota. Appl Environ Microbiol 70, 3575-3581.

Ben Omar, N. \& Ampe, F. (2000). Microbial community dynamics production of the Mexican fermented maize dough pozol. Appl Environ Microbiol 66, 3664-3673.
Bliss, D. Z., Guenter, P. A. \& Settle, R. G. (1992). Defining and reporting diarrhea in the tube-fed patients - what a mess! Am J Clin Nutr 55, 753-759.

Donskey, C. J. (2004). The role of the intestinal tract as a reservoir and source for transmission of nosocomial pathogens. Clin Infect Dis 39, 219-226.

Donskey, C. J., Hujer, A. M., Das, S. M., Pultz, N. J., Bonomo, R. A. \& Rice, L. B. (2003). Use of denaturing gradient gel electrophoresis for analysis of the stool microbiota of hospitalized patients. J Microbiol Methods 54, 249-256.

Giacomini, M., Borotto, E., Bosotti, L., Denkewitz, T., Reali-Foster, C., Carlucci, P., Centanni, S., Mantero, A. \& lapichino, G. (2007). Vardenafil and weaning from inhaled nitric oxide: effect on pulmonary hypertension in ARDS. Anaesth Intensive Care 35, 91-93.

Guarner, F. (2006). Enteric flora in health and disease. Digestion 73 (Suppl. 1), 5-12.

Guarner, F. \& Malagelada, J. R. (2003). Gut flora in health and disease. Lancet 361, 512-519.

He, J. W. \& Jiang, S. (2005). Quantification of enterococci and human adenoviruses in environmental samples by real-time PCR. Appl Environ Microbiol 71, 2250-2255.

Hebuterne, X. \& Schneider, S. M. (2001). Impact of enteral nutrition on gastrointestinal functions. Clin Nutr 20, 57-61.

Jernberg, C., Sullivan, A., Edlund, C. \& Jansson, J. K. (2005). Monitoring of antibiotic-induced alteration in the human intestinal microflora and detection of probiotic strains by use of terminal restriction fragment length polymorphism. Appl Environ Microbiol 71, 501-506.

Le Gall, J. R., Lemeshow, S. \& Saulnier, F. (1993). A new simplified acute physiology score (SAPS II) based on a European-North American multicenter study. JAMA 270, 2957-2963.

Mai, V. \& Morris, J. G., Jr (2004). Colonic bacterial flora: changing understandings in the molecular age. J Nutr 134, 459-464.

Marshall, J. C. (1999). Gastrointestinal flora and its alterations in critical illness. Curr Opin Clin Nutr Metab Care 2, 405-413.

O'Hara, A. M. \& Shanahan, F. (2006). The gut flora as a forgotten organ. EMBO Rep 7, 688-693.

Rakoff-Nahoum, S., Paglino, J., Eslami-Varzaneh, F., Edbergh, S. \& Medzhitov, R. (2004). Recognition of commensal microflora by tolllike receptors is required for intestinal homeostasis. Cell 118, 229-241.

Rinttila, T., Kassinen, A., Malinen, E., Krogius, L. \& Palva, A. (2004). Development of an extensive set of 16S rDNA-targeted primers for quantification of pathogenic and indigenous bacteria in faecal samples by real-time PCR. J Appl Microbiol 97, 1166-1177.

Scheppach, W., Muller, J. G., Boxberger, F., Dusel, G., Richter, F., Bartram, H. P., Christl, S. U., Dempfle, C. E. \& Kasper, H. (1997). Histological changes in the colonic mucosa following irrigation with short-chain fatty acids. Eur J Gastroenterol Hepatol 9, 163-168.

Schneider, S. M., Le Gall, P., Girard Pipau, E., Piche, T., Pompei, A., Nano, J. L., Hebuterne, X. \& Rampal, P. (2000). Total artificial nutrition is associated with major changes in the fecal flora. Eur $J$ Nutr 39, 248-255.

Seksik, P., Rigottier-Gois, L., Gramet, G., Sutren, M., Pochart, P., Marteau, P., Jian, R. \& Doré, J. (2003). Alterations of the dominant faecal bacterial groups in patients with Crohn's disease of the colon. Gut 52, 237-242.

Shimizu, K., Ogura, H., Goto, M., Asahara, T., Nomoto, K., Morotomi, M., Yoshiya, K., Matsushima, A., Sumi, Y. \& other authors (2006). Altered gut flora and enviroment in patients with severe SIRS. J Trauma 60, 126-133. 
Stiefel, U., Pultz, N. J., Helfand, M. S. \& Donskey, C. J. (2004). Increased susceptibility to vancomycin-resistant Enterococcus intestinal colonization persists after completion of anti-anaerobic antibiotic treatment in mice. Infect Control Hosp Epidemiol 25, 373-379.

Sullivan, A., Edlund, C. \& Nord, C. E. (2001). Effect of antimicrobial agents on the ecological balance of human microflora. Lancet Infect Dis 1, 101-114.

Tannock, G. W. (2002). Analysis of the intestinal microflora using molecular methods. Eur J Clin Nutr 56 (Suppl. 4), S44-S49.

Topping, D. L. \& Clifton, P. M. (2001). Short-chain fatty acids and human colonic function: roles of resistant starch and nonstarch polysaccharides. Physiol Rev 81, 1031-1064.

Vincent, J. L., Moreno, R., Takala, J., Willatts, S., De Mendonca, A., Bruining, H., Reinhart, C. K., Suter, P. M. \& Thijs, L. G. (1996). The SOFA (Sepsis-related Organ Failure Assessment) score to describe organ dysfunction/failure. On behalf of the Working Group on
Sepsis-Related Problems of the European Society of Intensive Care Medicine. Intensive Care Med 22, 707-710.

Walter, J., Tannock, G. W., Tilsala-Timisjarvi, A., Rodtong, S., Loach, D. M., Munro, K. \& Alatossava, T. (2000). Detection and identification of gastrointestinal Lactobacillus species by using denaturing gradient gel electrophoresis and species-specific PCR primers. Appl Environ Microbiol 66, 297-303.

Weber, S. G. \& Gold, H. S. (2003). Enterococcus: an emerging pathogen in hospitals. Semin Respir Crit Care Med 24, 49-60.

Young, V. B. \& Schmidt, T. M. (2004). Antibiotic-associated diarrhea accompanied by large-scale alterations in the compositions of the fecal microbiota. J Clin Microbiol 42, 1203-1206.

Zoetendal, E. G., Akkermans, A. D. L., Akkermans-van Vliet, W. M., de Visser, J. A. G. M. \& de Vos, W. M. (2001). The host genotype affects the bacterial community in the human gastrointestinal tract. Microb Ecol Health Dis 13, 129-134. 